OPEN ACCESS

University of Dundee

\title{
Substance misuse in patients who have comorbid chronic pain in a clinical population receiving methadone maintenance therapy for the treatment of opioid dependence
} Higgins, Cassandra; Smith, Blair; Matthews, Keith

Published in:

Drug and Alcohol Dependence

DOI:

10.1016/j.drugalcdep.2018.08.038

Publication date:

2018

Licence:

CC BY-NC-ND

Document Version

Peer reviewed version

Link to publication in Discovery Research Portal

Citation for published version (APA):

Higgins, C., Smith, B., \& Matthews, K. (2018). Substance misuse in patients who have comorbid chronic pain in a clinical population receiving methadone maintenance therapy for the treatment of opioid dependence. Drug and Alcohol Dependence, 193, 131-136. https://doi.org/10.1016/j.drugalcdep.2018.08.038

\section{General rights}

Copyright and moral rights for the publications made accessible in Discovery Research Portal are retained by the authors and/or other copyright owners and it is a condition of accessing publications that users recognise and abide by the legal requirements associated with these rights.

- Users may download and print one copy of any publication from Discovery Research Portal for the purpose of private study or research.

- You may not further distribute the material or use it for any profit-making activity or commercial gain.

- You may freely distribute the URL identifying the publication in the public portal.

Take down policy

If you believe that this document breaches copyright please contact us providing details, and we will remove access to the work immediately and investigate your claim. 


\section{OPEN ACCESS}

\section{University of Dundee}

\section{Substance misuse in patients who have comorbid chronic pain in a clinical population receiving methadone maintenance therapy for the treatment of opioid dependence}

Higgins, Cassandra; Smith, Blair; Matthews, Keith

Published in:

Drug and Alcohol Dependence

Publication date:

2018

Document Version

Peer reviewed version

Link to publication in Discovery Research Portal

Citation for published version (APA):

Higgins, C., Smith, B., \& Matthews, K. (Accepted/In press). Substance misuse in patients who have comorbid chronic pain in a clinical population receiving methadone maintenance therapy for the treatment of opioid dependence. Drug and Alcohol Dependence. 2018, 193 pp.131-136 Available 18 October 2018 https:// doi.org/10.1016/j.drugalcdep.2018.08.038 
(C) 2018. This manuscript version is made available under the CC-BY-NC-ND 4.0 license http:// creativecommons.org/licenses/by-nc-nd/4.0/

Alcohol Dependence

Elsevier Editorial System(tm) for Drug and Manuscript Draft

Manuscript Number: KK-18-1034R1

Title: Substance misuse in patients who have comorbid chronic pain in a clinical population receiving methadone maintenance therapy for the treatment of opioid dependence

Article Type: Full Length Article

Keywords: Opioid replacement therapy; Methadone maintenance therapy; Opioid dependence; Chronic pain; Illicit substance use.

Corresponding Author: Dr. Cassie Higgins, PhD

Corresponding Author's Institution: University of Dundee

First Author: Cassie Higgins, PhD

Order of Authors: Cassie Higgins, PhD; Blair H Smith, PhD; Keith Matthews, PhD

Abstract: Aims

To compare specific substance misuse in treatment-seeking, opioiddependent patients with and without comorbid chronic pain, and to assess the respective value of urinalysis and patient reports in assessing substance misuse.

Methods

Participants comprised a clinical population in a regional NHS Substance Misuse Service in the East of Scotland ( $N=521$ ). The Brief Pain Inventory - Short Form was used to assess pain and the Maudsley Addiction Profile and urinalysis were used to assess substance misuse at study inception. Urinalysis was used to assess substance misuse during the 5-year followup period. Data were hosted, linked, anonymised and analysed within a national Safe Haven.

Results

Compared with opioid-dependent patients with no pain, a significantly higher proportion of treatment-seeking, opioid-dependent patients with chronic pain were engaged in non-medical benzodiazepine use (69\% versus 58\%; $\mathrm{p}=0.016)$ and illicit cannabinoid use (84\% versus 65\%; p=0.025) at study inception. Furthermore, a significantly higher proportion of this group was shown to continue non-medical benzodiazepine use (70\% versus 42\%; $\mathrm{p}=0.037)$ and illicit cannabinoid use (100\% versus 32\%; $\mathrm{p}=0.002)$ during the 5-year follow-up period. There were significant correlations between drug screen results and patient-reported use of opioids (Tetrachoric $\mathrm{e}=0.4944 ; \mathrm{p}<0.001$ ), benzodiazepines (Tetrachoric $\mathrm{e}=0.2641$; $\mathrm{p}=0.001$ ) and cannabinoids (Tetrachoric $\mathrm{e}=0.8384 ; \mathrm{p}<0.001$ ).

Conclusions

Whilst gaining control of illicit opioid use during treatment, opioiddependent patients with comorbid chronic pain demonstrated persistent problematic use of benzodiazepines and cannabinoids. This pattern of misuse was shown to persist during the 5-year follow-up period. 
FULL TITLE: Substance misuse in patients who have comorbid chronic pain in a clinical population receiving methadone maintenance therapy for the treatment of opioid dependence.

RUNNING TITLE: Drug use in OAT patients with chronic pain.

AUTHORS: Cassie Higgins (corresponding author) ${ }^{a}$, Blair H Smith $^{b}$, Keith Matthews ${ }^{a}$

a Division of Neuroscience, University of Dundee, Mailbox 6, Level 6, Laboratories Block, Ninewells Hospital and Medical School, Dundee, DD1 9SY

${ }^{b}$ Division of Population Health Sciences, University of Dundee, Mackenzie Building, Kirsty Semple Way, Ninewells Hospital and Medical School, Dundee, DD2 4RB

Cassie Higgins, PhD. ORCID: 0000-0002-5506-324X Email: c.y.higgins@dundee.ac.uk

Blair H Smith, PhD. ORCID: 0000-0002-5362-9430. Email: $\underline{\text { b.h.smith@dundee.ac.uk }}$

Keith Matthews, PhD. ORCID: 0000-0002-4478-5888. Email: k.matthews@dundee.ac.uk

Colour required: No.

Word count: 3496 


\title{
Substance misuse in patients who have comorbid chronic pain in a clinical population receiving methadone maintenance therapy for the treatment of opioid dependence
}

\begin{abstract}
Aims

To compare specific substance misuse in treatment-seeking, opioid-dependent patients with and without comorbid chronic pain, and to assess the respective value of urinalysis and patient reports in assessing substance misuse.
\end{abstract}

Methods

Participants comprised a clinical population in a regional NHS Substance Misuse Service in the East of Scotland $(\mathrm{N}=521)$. The Brief Pain Inventory - Short Form was used to assess pain and the Maudsley Addiction Profile and urinalysis were used to assess substance misuse at study inception. Urinalysis was used to assess substance misuse during the 5-year follow-up period. Data were hosted, linked, anonymised and analysed within a national Safe Haven.

Results

Compared with opioid-dependent patients with no pain, a significantly higher proportion of treatment-seeking, opioid-dependent patients with chronic pain were engaged in non-medical benzodiazepine use (69\% versus $58 \% ; \mathrm{p}=0.016$ ) and illicit cannabinoid use ( $84 \%$ versus $65 \%$; $p=0.025$ ) at study inception. Furthermore, a significantly higher proportion of this group was shown to continue non-medical benzodiazepine use ( $70 \%$ versus $42 \%$; $p=0.037)$ and illicit cannabinoid use ( $100 \%$ versus $31 \% ; p=0.002$ ) during the 5 -year follow-up period. There were significant correlations 
between drug screen results and patient-reported use of opioids (Tetrachoric $\varrho=0.4944 ; p<0.001$ ), benzodiazepines (Tetrachoric $\varrho=0.2641 ; \mathrm{p}=0.001$ ) and cannabinoids (Tetrachoric $\varrho=0.8384 ; \mathrm{p}<0.001$ ).

Conclusions

Whilst gaining control of illicit opioid use during treatment, opioid-dependent patients with comorbid chronic pain demonstrated persistent problematic use of benzodiazepines and cannabinoids. This pattern of misuse was shown to persist during the 5 -year follow-up period.

Keywords: Opioid agonist therapy; methadone maintenance therapy; opioid dependence; chronic pain; and illicit substance use. 


\subsection{Introduction}

Chronic pain is highly prevalent in treatment-seeking, opioid-dependent populations with between 36\% and 68\% affected (Barry et al., 2013; Tsui et al., 2016). Thus, chronic pain is an important clinical condition to be considered by addiction specialists. Furthermore, patients in receipt of opioid agonist therapy (OAT) who have comorbid chronic pain are associated with relatively poor health and substance use treatment outcomes, further complicating the delivery of effective treatment in substance misuse services. This comorbid presentation is associated with a range of medical and psychiatric morbidities (Iskandar et al., 2013; O'Toole et al., 2013), in addition to relatively severe and enduring substance misuse problems (Dunn et al., 2014; Larson et al., 2007). In addressing this issue, many studies have focused on any substance misuse (rather than specific drug misuse) as the target variable (e.g. Caldeiro et al., 2008) or illicit opioid use versus any other substance misuse (Dennis et al., 2015); however, there is a need to understand specific drug misuse profiles in this comorbid population to identify any patterns or problems with specific substances. Only then can further research explore the potential causes of the high levels of drug misuse in this comorbid group and work towards effective treatment delivery in substance misuse services.

OAT programmes focus on a range of health-related and functional outcomes but the core outcomes are considered to be retention in treatment and control over substance use (Kidd et al., 2013). These two core aims are considered to lead to decreased mortality (Cousins et al., 2016), drugrelated harm reduction (Hutchinson et al., 2000; Nolan et al., 2014), increased health and functioning (Bart, 2012; Quednow \& Herdener, 2016), and crime reduction (Hutchinson et al., 2000; Russolillo et al., 2018). A number of studies have compared substance misuse outcomes in patients with and without comorbid chronic pain; however, they have tended to focus on any substance misuse, rather than attempting to profile use of specific substances (Caldeiro et al., 2008; Dennis et al., 2015). Barry and colleagues (2009) undertook a cross-sectional study examining illicit substance use in 150 methadone-maintained patients with and without clinically-significant pain (CSP). CSP 
was defined as pain having persisted for at least 6 months with moderate-severe intensity or significant pain interference. The control group reported no pain in the 7 days preceding assessment. The most commonly-reported substance misuse concerned illicit use of cocaine (25\%), cannabinoids (11\%), heroin (11\%), and nonmedical use of benzodiazepines (11\%); however, there were no significant group differences. In a more recent study, Dunn and colleagues (2014) examined the predictive capacity of pain on urinalyses in a methadone-maintained clinical population. Pain was significantly associated with mean percent of benzodiazepine-positive urine samples, but was not associated with opioid- or cocaine-positive samples.

A further concern in treatment-seeking, opioid-dependent populations is the extent of problematic substance use - the amount of drug consumption, duration of drug use and frequency of drug use. Trafton and colleagues (2004) examined frequency and duration of illicit substance use in a sample of 251 veterans attending eight ORT treatment facilities who were in receipt of either methadone or levo-alpha-acetyl-methadol (LAAM). They reported that, on entry to treatment, patients with pain had used illicit substances more frequently than patients with no pain in the preceding 30 days and for longer durations during their lifetimes. This finding related specifically to substances associated with potential analgesic effects. Increased frequency of illicit opioid used was noted in patients with pain in the preceding 30 days ( 2.3 versus 0.8 days in patients with no pain) and increased duration during their lifetimes (2.9 versus 0.9 years in patients with no pain). Increased frequency of illicit cannabinoid use was noted in patients with pain in the preceding 30 days ( 2.8 versus 0.8 days in patients with no pain) and increased duration during their lifetimes (10.3 versus 7.5 years in patients with no pain). Increased duration of nonmedical sedative use during their lifetimes was noted in patients with pain ( 2.5 versus 0.4 years in patients with no pain). There were no group associations with alcohol, cocaine, heroin or poly-substance use. Information concerning duration of pain symptoms were not available; therefore, one limitation of the study was that the authors were unable to distinguish between chronic and acute pain on entry to treatment. 
Whilst gaining a cross-sectional understanding of illicit substance use on entry to treatment is of some clinical relevance, examining response to OAT treatment in the longer term is of key importance to policy development and effective healthcare delivery. Caldeiro and colleagues (2008) examined abstinence at 12-month follow-up of 582 patients in a Veterans Affairs (VA) outpatient addiction treatment facility. Assessment of pain characteristics in the cohort resulted in the identification of three groups: low pain ( $n=114)$; intermittent pain $(n=275)$; and persistent pain $(n=193)$. The proportion of abstinence from illicit drug use was similar across all three groups at baseline; however, persistent pain was associated with a poorer outcome at 12-month follow-up. There was a significant stepwise relationship between pain group and abstinence at follow-up, with the highest proportion of abstinence found in the 'low pain' group (58\%) and the lowest proportion found in the 'persistent pain' group (44\%).

Effective management of substance misuse problems in patients with comorbid chronic pain is dependent upon understanding the particular profiles and the longer-term trajectories of drug misuse (Sheu et al., 2008). Several studies report 'long-term' follow-up over 12 months (e.g. Caldeiro et al., 2008; Ilgen et al., 2006). In this treatment group, however - which is characterised by problems that persist for years or even decades -12 months may be considered to be a relatively short duration. The aims of the present study are two-fold. First, to compare specific common drugs of misuse at study inception in methadone-maintained patients with and without chronic pain. Secondly, to compare misuse of the same substances in these two groups at 5-year follow-up. Continuation and initiation of illicit drug use were considered separately from each other since initiation of illicit drug use after commencing treatment may present different clinical challenges compared with continuation of illicit drug use despite treatment.

\subsection{Methods}




\subsubsection{Participants and setting}

Participants comprised methadone-maintained patients attending a National Health Service (NHS) substance misuse service in the East of Scotland. All participants were clinically-diagnosed as being dependent upon opioids - primarily heroin (as reported by the service) - on entry to treatment (using the World Health Organisation's International Classification of Diseases (ICD) diagnostic criteria), and many were also engaged in polysubstance abuse. A case-control design was employed; cases were treatment-seeking, opioid-dependent patients with comorbid chronic pain and controls were treatment-seeking, opioid-dependent patients with no pain. Three temporal thresholds have been established to identify chronic pain: 3 months; 6 months; and 12 months (Smith \& Torrance, 2008). In the present study the chronicity threshold was set at 12 months; the rationale for employing this threshold was that, in a clinical population familiar with persistent, debilitating conditions, the highest conventional threshold was considered to facilitate the best comparison between truly 'chronic' pain and no pain.

\subsubsection{Materials}

Sociodemographic data were collected at study inception using a proforma developed by the NHS treatment service. Data were collected using an interview format and the proforma recorded: age; gender; 3 accommodation-related questions; 7 questions concerning dependent children; 12 healthrelated questions; 3 education-related questions; and 2 questions concerning support from other agencies. Scores on the Scottish Index of Multiple Deprivation (SIMD) data were provided for all participants by the Safe Haven. Participants were then assigned to SIMD quintiles, whereby, Q1-Q2 represented relative deprivation and Q3-Q5 represented relative affluence.

A service-modified version of the Brief Pain Inventory - Short Form (BPI-SF; Cleeland \& Ryan, 1994) was used to identify and profile the severity and impact of pain at study inception. The BPI-SF is a 9item questionnaire intended to assess the sensory and reactive dimensions of pain. The BPI-SF has 
been validated in a number of clinical populations, including patients in receipt of methadone maintenance therapy for the treatment of opioid dependence (Dennis et al., 2016). One of the modifications was the addition of a question asking duration of pain (in months or years and months) at study inception.

A service-modified version of the Maudsley Addiction Profile (MAP; Marsden et al., 1998) was used to obtain information on substance misuse at study inception. The MAP is a 60-question, clinicianadministered instrument designed for research purposes and used in investigations of populations with drug and/or alcohol problems. Reliability and validity of the MAP have been demonstrated in substance misusers (Keary et al., 2012).

An electronic regional extract of the nationally-held NHS Scotland biochemical laboratory test dataset provided drug screen results. This dataset was used to identify all positive and negative results for the presence in the urine of opioids, benzodiazepines and cannabinoids (the three most commons drugs of abuse in this particular treatment service).

\subsubsection{Procedure}

The study was incepted at the beginning of 2005 , and follow-up data were collected at routine intervals during the five year follow-up period (2005-2010). The BPI-SF instruments were completed at routine weekly clinic appointments with specialist addiction nurses at study inception. Staff were trained on administration of these instruments, to ensure uniformity in data collection procedures. Participants were excluded from the study if no BPI-SF was completed or if duration of pain at study inception was not recorded, since it was not possible to determine if their pain was 'chronic'.

Urine samples were intended to be collected 3-monthly during the follow-up period and underwent biochemical testing. The follow-up biochemical data were dependent, however, upon urine samples 
having been obtained in the clinic setting. The NHS treatment service aims to undertake drug screens, by urinalysis, every three months with each patient. The amount of missing data indicates that this was not achieved for all patients. Urinalysis is associated with a relatively high risk of falsepositive and false-negative results (Marsden et al., 1998; Nelson et al., 2016; Riahi-Zanjani, 2014; Saitman et al., 2014). In the present study, therefore, illicit drug use was concluded where there were at least three positive test results in a 12-month period. These data were obtained for the 12 month period at study inception (calendar year 2005) and the 12-month period at 5-year follow-up (calendar year 2010).

MAP data were obtained at study inception only, during routine weekly clinic appointments with specialist addiction nurses at study inception. Staff were trained on the administration of these instruments, to ensure uniformity in data collection procedures. Substance misuse data were extracted from the MAP in binary form so as to facilitate comparisons with the urinalysis findings.

Biochemical laboratory data were obtained and hosted by one of the national Safe Havens, where they were electronically-linked to the data collected at the NHS treatment service. All data were anonymised within the Safe Haven prior to release to the research team and statistical analyses were undertaken within this secure virtual environment.

\subsubsection{Statistical considerations}

The Statistical Package for Social Scientists (SPSS v22) was used to undertake statistical testing. Statistical findings are reported as chi-square value and degrees of freedom ( $\chi 2(\mathrm{df})$ ), probability value ( $p)$ and effect size, Pearson's Phi or Cramer's V $(\omega)$. Pearson's Phi was used to assess the effect size in $2 \times 2$ contingency tables and Cramer's $V$ was used where there were more than two levels in independent variables. Descriptive summary data are presented as number of event ( $\mathrm{n}$ ) and percentage of group (\%). For univariate analysis of variance (ANOVA) findings are reported as $F$ 
value, between-subjects degrees of freedom and within-subjects degrees of freedom ( $F($ betweensubjects df, within-subjects df)), probability value $(p)$ and effect size, partial eta squared $\left(\eta_{p}^{2}\right)$. Descriptive summary data are presented as mean value $(x)$ and standard deviation around the mean (б). Maximum likelihood Tetrachoric correlation coefficients were undertaken, using Stata v14, to provide rho estimates of the correlation between patient-reported illicit substance use and urinalysis results. Findings are reported as the Tetrachoric rho $(\varrho)$, the standard error $(\sigma)$ and the probability value $(p)$.

\subsubsection{Ethical considerations}

Ethical approval was not required for the present study, since all data were anonymised and accessed via a national Safe Haven; however, a favourable ethical opinion was obtained from the East of Scotland Research Ethics Committee (EOSREC).

\subsection{Results}

\subsubsection{Representativeness of study cohort}

Assessment of demographic, socioeconomic, educational, health and personal characteristics revealed that the study cohort $(n=521)$ was broadly representative of the entire treatment population $(n=626)$. A significantly greater proportion of those that were excluded due to missing data comprised patients that were: relatively affluent; living in urban locations; and associated with fewer physical health problems.

1.3.2 Group identification, clinical profile of chronic pain and therapeutic methadone dose at study inception

Of the 521 patients that completed a full pain assessment, 54 (11\%) were excluded from analysis as they reported pain duration of less than 12 months. Just over half of the remainder ( $n=246 ; 53 \%)$ 
were identified as having comorbid chronic pain (cases, referred to as the 'CP group'), whilst 221 (47\%) reported no pain at study inception (controls, referred to as the 'NoP group'). Not all participants answered all questions on the BPI-SF and, in consequence, percentages were calculated in respect of the number having responded to each question. Patients with chronic pain reported a mean duration of pain of 99 months ( $S D=88$; median=72; range=12-550). On a $0-100$ scale these patients reported a mean pain intensity of $63(S D=21$; median=65; range=4-100). Almost a quarter $(24 \%, n=58)$ reported pain at multiple sites (i.e. two or more sites), $70 \%(n=173)$ reported pain interference in daily activities and $80 \%(n=197)$ reported sleep interference as a consequence of pain. More than three quarters $(79 \%, n=175)$ had attended a physician for treatment of their pain and, of those, $41 \%(n=75)$ were treated by a pain specialist. Just over half of those treated by a physician $(59 \%, n=113)$ felt that their pain problem had been taken seriously. Just over half $(56 \%$, $n=114)$ were in receipt of an analgesic prescription with $22 \%(n=45)$ of them having been in receipt of an opioid analgesic prescription, in addition to methadone prescribed as OAT for the treatment of opioid dependence. The CP group was in receipt of a significantly higher methadone dose of methadone $\left(F(1,419)=4.370 ; \mathrm{p}=0.037\left(\eta_{p}^{2}=0.010\right)\right)$. The mean daily dose of the $\mathrm{CP}$ group (55mg; $\sigma=24$ ) was more than double that of the NoP group (25mg; $\sigma=25$ ).

\subsubsection{Demographic and socioeconomic characteristics of patients with chronic pain and without pain} at study inception

The two groups had very similar demographic profiles. Almost three quarters of each group was male and around $90 \%$ of each group was living in areas of high socioeconomic deprivation (SIMD quintiles 1 and 2). The CP group was significantly older (35 years; SD=7.5) than the NoP group (32 years; $S D=7.9)$ by a mean difference of just under 3 years $(F(1,428)=14.980 ; p<0.001)$.

\subsubsection{Illicit substance use in patients with and without chronic pain at study inception}


Misuse of opioids, benzodiazepines and cannabinoids is shown in Table 1. Patient-reported use is shown in the first half of the table and urinalysis results are shown in the second half of the table. Patient-reported illicit opioid use included heroin, methadone and other opioid analgesics; however, in consequence of all patients having been in receipt of prescription methadone, biochemical drug screens for illicit opioid use did not include screening for methadone.

[Insert Table 1 around here]

Table 1 shows that there was no significant difference between groups in their use of opioids with more than half of each group reporting illicit use. A statistically significant correlation was found between patient-reported illicit opioid use and urinalysis results (Tetrachoric $\varrho=0.4944 ; \sigma=0.0636$; $\mathrm{p}<0.0001)$. Urinalysis identified slightly fewer positive results than expected, based on patient reports.

There was a statistically significant correlation found between patient-reported illicit benzodiazepine use and urinalysis results (Tetrachoric $\varrho=0.2641 ; \sigma=0.0770 ; p=0.0011$ ). Patient reports indicated no group difference with around a third of each group reporting illicit use of benzodiazepines. There were, however, many who denied nonmedical benzodiazepine use, despite this having been found on urinalysis, particularly in the CP group. Urinalysis revealed that a significantly higher proportion of the CP group generated positive benzodiazepine results.

There was a statistically significant correlation found between patient-reported illicit cannabinoid use and urinalysis results (Tetrachoric $\varrho=0.8384 ; \sigma=0.0736 ; p<0.0001$ ). Both patient reports and urinalyses revealed that a significantly higher proportion of the CP group was engaging in illicit cannabinoid use. 


\subsubsection{Illicit substance use during the 5-year follow-up period}

A significantly higher proportion of the CP group ( $n=160 ; 65 \%$ ) was retained in treatment compared with the NoP group ( $n=105 ; 48 \%$ ). Of those not retained, a significantly higher proportion of the CP group $(n=26 ; 30 \%)$ was deceased at follow-up compared with the NoP group $(n=13 ; 11 \%)$. The survivor bias (or 'survivor effect') should be borne in mind when interpreting the remainder of the findings, since the patients that died would have been likely to have had more problems and poorer health. The effect of the survivor bias, in this context, would be an underestimation of problems and an overestimation of wellbeing, particularly in the CP group. In consequence, there would be a muting of group differences in cases where the CP group was associated with poorer outcomes.

Chi square was used to analyse change in results between study inception and follow-up. Continuation and initiation of illicit drug use were considered separately from each other since initiation of illicit drug use after commencing treatment may present different clinical challenges compared with continuation of illicit drug use despite treatment. Continuation of illicit drug use was compared to cessation during the follow-up period, and initiation of illicit drug use was compared to no use at study inception or at follow-up. Findings are reported in Table 2.

[Insert Table 2 around here]

Table 2 shows that a significantly higher proportion of the NoP group than the CP group continued illicit opioid use during the 5-year follow-up period; however, there was no significant group difference in the initiation of illicit opioid use during the follow-up period. Conversely, a significantly higher proportion of the $\mathrm{CP}$ group than the NoP group continued use of benzodiazepines and cannabinoids. There was no significant group difference in the initiation of non-medical benzodiazepine use and there were insufficient data to test for group differences in the initiation of illicit cannabinoid use during the follow-up period. 


\subsection{Discussion}

The aim of the present study was to compare problematic substance use in treatment-seeking, opioid-dependent patients with and without comorbid chronic pain. Comparisons were undertaken at study inception and at 5-year follow-up. The prevalence of chronic pain in this population in receipt of OAT was found to be $53 \%$. There were no differences between those with and those without chronic pain in their illicit opioid use at study inception; however, significantly higher proportions of the group with comorbid chronic pain were engaged in non-medical benzodiazepine use and illicit use of cannabinoids. Continuation of illicit opioid use was significantly elevated in the group with no pain, whilst continuation of benzodiazepine and cannabinoid use was significantly elevated in the comorbid pain group.

Biochemical drug screen results are generally preferred to patient-reported drug use (Li et al., 2017); however, there are advantages associated with patient-reported data. First, it is possible to obtain information concerning the amount used and the routes of administrations. Secondly, in methadone-maintained populations, due to the need to control for prescribed methadone, it is not possible to include methadone in screens for illicit opioid use. Indeed, in the present study, urinalysis identified slightly fewer positive results than expected, based on patient reports, and this disparity may be due to the absence of methadone screens. The general preference for biochemical drug screen results is based on concerns around the respective validity of the two methods of data collection, as discussed by $\mathrm{Li}$ and colleagues (2017). Similar to the findings of their study, the present study found statistical correlations between patient reports and urinalysis findings for all drugs examined. This finding requires further replication, which may highlight the potential value of the inclusion of patient-reported drug use in future studies. 
A significantly higher proportion of the group with no pain reported illicit heroin use; however, this finding was not corroborated by opioid drug screen results. This may be a result of urinalysis not screening, specifically, for heroin; therefore, opioid screens also included illicit use of opioid analgesics, but not methadone. Alternatively, this finding may be an artefact of the short half-life of heroin ( $\sim 30$ minutes for a single dose) and the time between drug use and testing, resulting in patient-reported heroin use but negative urinalysis findings, further highlighting the potential value of patient-reported data. A significantly higher proportion of this group continued illicit opioid use during the follow-up period. Heroin is the primary drug of abuse in most patients entering this particular OAT programme and, as such, is the principal drug of concern when assessing response to treatment. Furthermore, negative opioid drug screens are often a requirement in OAT programmes for continued treatment delivery. This finding may reflect efforts in the comorbid pain group to conform to treatment agreements or it may reflect relative instability in patients with no pain.

Significantly higher proportions of the comorbid pain group were engaged in nonmedical benzodiazepine use and illicit cannabinoid use at study inception, and significantly higher proportions continued use of both drugs during the follow-up period. The denial of urinalysisverified benzodiazepine use may reflect substantial use of benzodiazepines other than diazepam or may reflect efforts to conceal nonmedical use of benzodiazepines in both groups, but particularly in the comorbid pain group. Benzodiazepines are frequently used in conjunction with heroin or other opioids to intensify the euphoric experience (Jones et al., 2012); as such, benzodiazepine use may be viewed as part of the profile of 'problematic drug use' and patients may feel a need to conceal its use. This finding highlights the value in using objective measures of substance use to corroborate patient reports. Alternatively, misuse of these drugs may reflect attempts to control unmanaged symptoms. The analgesic properties of cannabinoids are well-documented (Elikottil et al., 2009; Lotsch et al., 2018; Manzanares et al., 2006), and benzodiazepines can be used as an adjunct to analgesia or as a muscle relaxant as part of a pain management regimen. As such, patients may use 
these drugs to control unmanaged pain. Furthermore, this profile of drug use is commonly associated with patients with psychiatric problems (Brunette et al., 2003; Diehl et al., 2010; Grattan et al., 2012). Psychiatric morbidity is known to be highly prevalent in both opioid-dependent patients (Ardakani et al., 2013; Roncero et al., 2016) and in patients with pain problems (de Heer et al., 2014; Woo, 2010) and, therefore, may be amplified in comorbid populations (Dhingra et al., 2013; Fox et al., 2012). These findings may reflect under-treatment for psychiatric distress which results in 'chaotic' lifestyles and behaviours and, in opioid-dependent patients, this may manifest as uncontrollable substance use problems. Future studies should consider the role of pain and psychiatric morbidity when examining problematic substance use in opioid-dependent populations.

\subsubsection{Limitations}

A key limitation of the present study is that pain assessments were undertaken at study inception only and not at follow-up. This may have resulted in a degree of misclassification at follow-up, since some patients may have developed chronic pain during the observation period and, indeed, some may have recovered. Furthermore, as a result of the limited statistical tests available for analysing binary data, only two time-points were observed - study inception and 5-year follow-up. It would have been interesting to have been able to compare across each of the years during the observation period. Our definition of illicit drug use (three positive drug tests in a 12-month period) was conservative to avoid misclassification arising through false positive tests. A less stringent definition would have identified higher proportions, but would also likely have included some who were identified inappropriately. There is no agreed definition of illicit drug use based on urine testing, and ours may have resulted in the under-identification of drug misuse. It should be noted that the data are 8 years old. Whilst they are likely to be relevant currently, it is possible that drug use profiles may have changed slightly due to drug availability in this specific geographic location. Finally, there was a significant group difference in terms of age; whilst this may represent statistical significance, the mean difference of almost three years may be considered to be of minimal clinical relevance. 


\subsubsection{Conclusions}

Patients attending an OAT service who have chronic pain were shown to be more likely to misuse benzodiazepines and cannabinoids, but not opioids, than those without pain. This pattern of drug use was shown to persist in this group over the 5-year follow-up period. The role of unmanaged pain and psychiatric morbidity should be examined further, and future studies should acknowledge the potential corroborative and elucidation value of using both patient reports and drug screen results when assessing substance misuse. 


\section{References}

Ardakani SMY, Banaei-Boroujeni S, Dastjerdi G (2013) Psychiatric Disorders Prevalence Comparison in Opiate-Dependent and Non-Opiate Dependent Individuals. J Addict Res Ther. Open Access DOI: 10.4172/2155-6105.S8-005. Accessed April 2018.

Barry DT, Beitel M, Garnet B, Joshi D, Rosenblum A, Schottenfeld RS (2009) Relations among psychopathology, substance use, and physical pain experiences in methadone-maintained patients. J Clin Psychiatry, 70(9):1213-1218.

Barry DT, Savant JD, Beitel M, Cutter CJ, Moore BA, Schottenfeld RS, Fiellin DA (2013) Pain and associated substance use among opioid dependent individuals seeking office-based treatment with buprenorphine-naloxone: a needs assessment study. Am J Addict, 22(3):212-217.

Bart G (2012) Maintenance Medication for Opiate Addiction: The Foundation of Recovery. J Addict Dis, 31(3): 207-225.

Brunette MF, Noordsy DL, Xie H, Drake RE (2003) Benzodiazepine use and abuse among patients with severe mental illness and co-occurring substance use disorders. Psychiatr Serv, 54(10):1395401.

Caldeiro RM, Malte CA, Calsyn DA, Baer JS, Nichol P, Kivlahan DR, Saxon AJ (2008) The association of persistent pain with out-patient addiction treatment outcomes and service utilization. Addiction, 103:1996-2005.

Cleeland CS, Ryan KM (1994) Pain assessment: global use of the Brief Pain Inventory. Ann Acad Med Singapore, 23(2):129-138. 
Cousins G, Boland F, Courtney B, Barry J, Lyons S, Fahey T (2016) Risk of mortality on and off methadone substitution treatment in primary care: a national cohort study. Addiction, 111(1):73-82.

de Heer EW, Gerrits MMJG, Beekman ATF, Dekker J, van Marwijk HWJ, de Waal MWM, Spinhoven P, Penninx BWJH, van der Feltz-Cornelis CM (2014) The Association of Depression and Anxiety with Pain: A Study from NESDA. PLoS One, 9(10): e106907.

Dennis BD, Roshanov PS, Bawor M, Paul J, Varenbut M, Daiter J, Plater C, Pare G, Marsh DC, Worster A, Desai D, Thabane L, Samaan Z (2016) Usefulness of the Brief Pain Inventory in Patients with Opioid Addiction Receiving Methadone Maintenance Treatment. Pain Physician, 19:E181-E195.

Dennis BB, Monica M, Naji L, Chan CK, Varenbut J, Paul J, Varenbut M, Daiter J, Plater C, Pare G, Marsh DC, Worster A, Desai D, Thabane L, Samaan Z (2015) Impact of Chronic Pain on Treatment Prognosis for Patients with Opioid Use Disorder: A Systematic Review and Meta-analysis. Subst Abuse, 9:59-80.

Dhingra L, Masson C, Perlman DC, Seewald RM, Katz J, McKnight C, Homel P, Wald E, Jordan AE, Young C, Portenoy RK (2013) Epidemiology of pain among outpatients in methadone maintenance treatment programs. Drug Alcohol Depend, 128(1-2):161-165.

Diehl A, Cruz Cordeiro D, Laranjeira R (2010) Cannabis abuse in patients with psychiatric disorders: an update to old evidence. Rev Bras Psiquiatr, 32, Suppl 1:S41-5.

Dunn KE, Brooner RK, Clark MR (2014) Severity and Interference of Chronic Pain in Methadone-Maintained Outpatients. Pain Med, 15(9):1540-1548. 
Elikottil J, Gupta P, Gupta K (2009) The Analgesic Potential of Cannabinoids. J Opioid Manag, 5(6): 341-357.

Fox AD, Sohler NL, Starrels JL, Ning Y, Giovanniello A, Cunningham CO (2012) Pain is not associated with worse office-based buprenorphine treatment outcomes. Subst Abus, 33(4):361-365.

Grattan A, Sullivan MD, Saunders KW, Campbell Cl, Von Korff MR (2012) Depression and Prescription Opioid Misuse Among Chronic Opioid Therapy Recipients With No History of Substance Abuse. Ann Fam Med, 10(4):304-311.

Hutchinson SJ, Taylor A, Gruer L, Barr C, Mills C, Elliott L (2000) One-year follow-up of opiate injectors treated with oral methadone in a GP-centred programme. Addiction, 95(7):1055-1068.

Ilgen MA, Trafton JA, Humphreys K (2006) Response to methadone maintenance treatment of opiate dependent patients with and without significant pain. Drug Alcohol Depend, 82(3):187-193.

Iskandar S, van Crevel R, Hidayat T, Siregar IMP, Achmad TH, van der Ven AJ, Do Jong CA (2013) Severity of psychiatric and physical problems is associated with lower quality of life in methadone patients in Indonesia. Am J Addict, 22(5):425-431.

Jones JD, Mogali S, Comer SD (2012) Polydrug abuse: A review of opioid and benzodiazepine combination use. Drug Alcohol Depend, 125(1-2): 8-18. 
Keary CJ, Wang Y, Moran JR, Zayas LV, Stern TA (2012) Toxicologic Testing for Opiates: Understanding False-Positive and False-Negative Test Results. Prim Care Companion CNS Disord, 14(4): PCC.12f01371.

Kidd BA, Lind C, Roberts K (2013) 'Delivering Recovery'. Edinburgh: Scottish Government.

Larson MJ, Passche M, Cheng DM, Lloyd-Travaglini C, Saitz R, Samet JH (2007) Persistent pain is associated with substance use after detoxification: a prospective cohort analysis. Addiction, 102(5):752-760.

Li L, Liang L-J, Lin C, Wu Z (2017) Comparison Between Urinalysis Results and Self-Reported Heroin Use Among Patients Undergoing Methadone Maintenance Treatment in China. Subst Use Misuse, 52(10): 1307-1314

Lotsch J, Weyer-Menkhoff I, Tegeder I (2018) Current evidence of cannabinoid-based analgesia obtained in preclinical and human experimental settings. Eur J Pain, 22(3): 471-484.

Manzanares J, Julian MD, Carrascosa A (2006) Role of the Cannabinoid System in Pain Control and Therapeutic Implications for the Management of Acute and Chronic Pain Episodes. Curr Neuropharmacol, 4(3): 239-257.

Marsden J, Gossop G, Stewart D, Best D, Farrell M, Lehmann P, Edwards C, Strang J (1998) The Maudsley Addiction Profile (MAP): A brief instrument for assessing treatment outcome. Addiction, 93(12):1857-1867. 
Nelson ZJ, Stellpflug SJ, Engebretsen KM (2016) What Can a Urine Drug Screening Immunoassay Really Tell Us? J Pharm Pract, 29(5):516-526.

Nolan S, Dias Lima V, Fairbairn N, Kerr T, Montaner J, Grebely J, Wood E (2014) The impact of methadone maintenance therapy on hepatitis $\mathrm{C}$ incidence among illicit drug users. Addiction, 109(12):2053-2059.

O'Toole J, Hambly R, Cox A-M, O'Shea B, Darker C (2013) Methadone-maintained patients in primary care have higher rates of chronic disease and multimorbidity, and use health services more intensively than matched controls. Eur J Gen Pract, 20(4):275-280.

Quednow BB, Herdener M (2016) Chapter 11 - Human pharmacology for addiction medicine: From evidence to clinical recommendations. Prog Brain Res, 224: 227-250.

Riahi-Zanjani B (2014) False Positive and False Negative Results in Urine Drug Screening Tests: Tampering Methods and Specimen Integrity Tests. Pharmacology Online, 1:102-108.

Roncero C, Barral C, Rodríguez-Cintas L, Pérez-Pazos J, Martinez-Luna N, Casas M, Torrens M, GrauLópez L (2016) Psychiatric comorbidities in opioid-dependent patients undergoing a replacement therapy programme in Spain: The PROTEUS study. Psychiatry Res, 243:174-81.

Russolillo A, Moniruzzaman A, McCandless LC, Patterson M, Somers JM (2018) Associations between methadone maintenance treatment and crime: a 17-year longitudinal cohort study of Canadian provincial offenders. Addiction, 113(4):656-667. 
Saitman A, Park HD, Fitzgerald RL (2014) False-positive interferences of common urine drug screen immunoassays: a review. J Anal Toxicol, 38(7):387-396.

Sheu R, Lussier D, Rosenblum A, Fong C, Portenoy J, Joseph H, Portenoy RK (2008) Prevalence and Characteristics of Chronic Pain in Patients Admitted to an Outpatient Drug and Alcohol Treatment Program. Pain Med, 9(7): 911-917.

Smith BH, Torrance N. "Epidemiology of Chronic Pain", in McQuay HJ, Kalso E, Moore RA (eds): Systematic Reviews in Pain Research: Methodology Refined. Seattle: IASP Press 2008, pp247 - 273.

Trafton JA, Oliva EM, Horst DA, Minkel JD, Humphreys K (2004) Treatment needs associated with pain in substance use disorder patients: implications for concurrent treatment. Drug Alcohol Depend, 73(1):23-31.

Tsui JI, Lira MC, Cheng DM, Winter MR, Alfordb DP, Liebschutz JM, Edwards RR, H.Samet JH (2016) Chronic pain, craving, and illicit opioid use among patients receiving opioid agonist therapy. Drug Alcohol Depend, 166: 26-31.

Woo AKM (2010) Depression and Anxiety in Pain. Rev Pain, 4(1): 8-12. 
Table 1: Group differences in patient-reported substance use in the 28 days prior to study inception and biochemical drug screen results at study inception. [Prescription medication was controlled in analyses of biochemical drug screen results.]

\begin{tabular}{|c|c|c|c|c|}
\hline & \multicolumn{2}{|c|}{ CP } & \multicolumn{2}{|c|}{ NoP } \\
\hline & $\mathbf{N}$ & $\%$ & $\mathbf{N}$ & $\%$ \\
\hline Patient-reported illicit opioid use & \multicolumn{4}{|c|}{$\chi^{2}(1)=1.814 ; p=0.178(\omega=0.067)$} \\
\hline Yes & 111 & 52 & 110 & 59 \\
\hline No & 102 & 48 & 77 & 41 \\
\hline Patient-reported illicit heroin use & \multicolumn{4}{|c|}{$\chi^{2}(1)=3.283 ; p=0.044(\omega=0.090)$} \\
\hline Yes & 86 & 40 & 92 & 49 \\
\hline No & 128 & 60 & 95 & 51 \\
\hline Patient-reported illicit diazepam use & \multicolumn{4}{|c|}{$\chi^{2}(1)=0.029 ; p=0.475(\omega=0.008)$} \\
\hline Yes & 73 & 34 & 62 & 33 \\
\hline No & 142 & 66 & 125 & 67 \\
\hline Patient-reported illicit cannabis use & \multicolumn{4}{|c|}{$\chi^{2}(1)=8.037 ; p=0.003(\omega=0.142)$} \\
\hline Yes & 172 & 81 & 128 & 68 \\
\hline No & 41 & 19 & 59 & 32 \\
\hline Positive biochemistry opioid results & \multicolumn{4}{|c|}{$\chi^{2}(1)=2.537 ; p=0.067(\omega=0.076)$} \\
\hline Yes & 106 & 47 & 114 & 55 \\
\hline No & 120 & 53 & 95 & 45 \\
\hline Positive biochemistry benzodiazepine results & \multicolumn{4}{|c|}{$\chi^{2}(1)=5.062 ; p=0.016(\omega=0.108)$} \\
\hline Yes & 153 & 69 & 121 & 58 \\
\hline No & 70 & 31 & 87 & 42 \\
\hline Positive biochemistry cannabinoid results & \multicolumn{4}{|c|}{$\chi^{2}(1)=4.720 ; p=0.025(\omega=0.210)$} \\
\hline Yes & 46 & 84 & 34 & 65 \\
\hline No & 9 & 16 & 18 & 35 \\
\hline
\end{tabular}


Table 2: Changes in urine drug screen results between study inception and 5-year follow-up.

\begin{tabular}{|c|c|c|c|c|}
\hline \multirow[t]{2}{*}{ Urine biochemistry results for illicit substance use } & \multicolumn{2}{|c|}{$\mathbf{C P}$} & \multicolumn{2}{|c|}{ NoP } \\
\hline & $\mathbf{N}$ & $\%$ & $\mathbf{N}$ & $\%$ \\
\hline Opioids: positive results at inception & \multicolumn{4}{|c|}{$\chi^{2}(1)=5.237 ; p=0.027(\omega=0.411)$} \\
\hline Continuation of use during the follow-up period & 5 & 36 & 13 & 77 \\
\hline Cessation of use during the follow-up period & 9 & 64 & 4 & 23 \\
\hline Benzodiazepines: positive results at inception & \multicolumn{4}{|c|}{$\chi^{2}(1)=4.268 ; p=0.037(\omega=0.289)$} \\
\hline Continuation of use during the follow-up period & 19 & 70 & 10 & 42 \\
\hline Cessation of use during the follow-up period & 8 & 30 & 14 & 58 \\
\hline Cannabinoids: positive results at inception & \multicolumn{4}{|c|}{$\chi^{2}(1)=9.692 ; p=0.002(\omega=0.679)$} \\
\hline Continuation of use during the follow-up period & 8 & 100 & 4 & 31 \\
\hline Cessation of use during the follow-up period & 0 & 0 & 9 & 69 \\
\hline Opioids: negative results at inception & \multicolumn{4}{|c|}{$\chi^{2}(1)=0.134 ; p=0.459(\omega=0.047)$} \\
\hline Initiation of use during the follow-up period & 15 & 45 & 11 & 41 \\
\hline No use at study inception or follow-up & 18 & 55 & 16 & 59 \\
\hline Benzodiazepines: negative results at inception & \multicolumn{4}{|c|}{$\chi^{2}(1)=2.694 ; p=0.103(\omega=0.290)$} \\
\hline Initiation of use during the follow-up period & 14 & 78 & 7 & 50 \\
\hline No use at study inception or follow-up & 4 & 22 & 7 & 50 \\
\hline Cannabinoids: negative results at inception & \multicolumn{4}{|c|}{ Not computed } \\
\hline Initiation of use during the follow-up period & 0 & 0 & 0 & 0 \\
\hline No use at study inception or follow-up & 2 & 100 & 1 & 100 \\
\hline
\end{tabular}




\section{Author Disclosure Statement}

\section{Role of Funding Source}

Whilst the present study received no funding, the data used in its preparation were obtained from a previously-conducted study. The funding for that study was obtained from Scottish Government monies that had been ring-fenced for the improvement of substance misuse services, directed by the regional Alcohol and Drug Partnerships (ADPs).

\section{Acknowledgements}

We would like to acknowledge the support of Dr Brian A Kidd in sharing his data; thereby, making this study possible. 
${ }^{\star}$ Conflict of interest statement

\section{Conflict of Interest}

No conflict declared. 


\section{Contributors}

All three authors contributed to the conceptual design and the production of the manuscript. $\mathrm{CH}$ undertook all statistical analyses. All authors have approved the final article. 


\section{Highlights}

- Opioid-dependent patients with chronic pain are more likely to misuse benzodiazepines

- Opioid-dependent patients with chronic pain are more likely to misuse cannabinoids

- Opioid-dependent patients with chronic pain are not more likely to misuse opioids

- This pattern of substance misuse is shown to persist in the longer term 


\section{RESPONSE TO EDITOR}

Manuscript number: KK-18-1034

Resubmission deadline: 20 October 2018

\section{Reviewer \#1}

Despite the relevance of this paper, I think there are several areas that could be improved. One involves the use of dichotomous outcomes (yes/no) for several outcomes, including the primary ones, even though it is specified in the methods the MAP gives information on quantity and frequency of substance use. If the order of the data lies in dichotomous outcome, then I think this is fine, but some rationale should be given in the methods as to why the data were analyzed in this manner. One question I had as I was reading the primary findings was if there was any difference in frequency of any of these substances from inception to 5-year follow up.

RESPONSE: Yes, the MAP produces data on frequency and quantity of drug use in addition to making these data available in the binary format that we used in the present manuscript. We have now removed the sentence referred to in the above comment, since it adds little to the manuscript. We have now also described, in the Methods>Procedure section, why we chose to use the binary data (i.e. to enable comparison with the biochemical drug screens, which were recorded as either positive or negative results). As part of the overall project, we did examine frequency of use, as suggested by this reviewer; however, due to space limitations, we had to be selective in what we included in the manuscript. We believe that presence/absence of misuse provides a better overall picture of the extent of problematic use in these groups.

The results section is devoted largely to discussing representativeness and demographic variables, but these are not discussed at any length in the discussion. Thus, there is a lot of information presented that should either be discussed or reduced to highlight the primary outcomes of this study. Also, the tables were hard to follow. Some reorganization could be helpful.

RESPONSE: We have now reduced these sections so as to highlight the primary outcomes of the present study. In reducing these sections, the tables were also removed. Each section now contains only a brief paragraph overviewing the key variables. 
There was no mention of why data from 2005-2010 were used. My guess is that these data/findings are still relevant, but I think noting that the data are 8-years-old in the limitations and how any of these issues may have changed since 2010 is important.

RESPONSE: We have now acknowledged this as a limitation, and explained that, whilst these data are likely to remain relevant, it is possible that changes have occurred in drug use profiles as a result of drug availability in the specific geographic location.

\section{Reviewer \#4}

Although the questions raised by the authors are important and clinically relevant, I believe that several important references are missing from the manuscript. Moreover, clarification of several methodological issues is necessary before the manuscript is considered for publication. Last, the authors should consider editing the Discussion so that more time is spent on issues that are more relevant to the data presented in the present manuscript.

General Comments

1.) The authors use the term opioid replacement therapy throughout the manuscript. I believe the preferred term is now opioid agonist therapy (OAT). The authors should consider making this change throughout the document.

RESPONSE: As suggested, this has now been changed throughout the manuscript.

2.) The authors should carefully proofread the document. The spacing after periods is inconsistent (particularly in the Results section). Furthermore, multiple periods are used in the first paragraph of the Results.

RESPONSE: Extraneous periods have now been removed and there are two spaces following every period (excepting those at the end of paragraphs and those in the References section). 
Abstract

3.) In the abstract, the authors report that $32 \%$ of the no pain group continued illicit cannabinoid use during the 5-year follow-up period; however, $31 \%$ is reported in Table 4. This is likely just an oversight; however, the manuscript should be carefully reviewed to ensure that all numbers are correct.

RESPONSE: Thank you for drawing our attention to this; it was, indeed, an oversight. We have now carefully reviewed the manuscript again to ensure that there are no other typographical errors.

Introduction

4.) The authors cite Jamison et al., $\mathbf{2 0 0 0}$ for an estimate of the frequency of chronic pain among opioid-dependent individuals. I think more recent estimates are available in the following articles:

a. Barry et al., 2013 Pain and Associated Substance Use among Opioid Dependent Individuals Seeking Office-Based Treatment with Buprenorphine-Naloxone: A Needs Assessment Study

b. Tsui et al., 2016 Chronic pain, craving, and illicit opioid use among patients receiving opioid agonist therapy

RESPONSE: Thank you for drawing this to our attention; these references have now been cited in order to provide more up-to-date estimates.

5.) The authors make the claim that comorbid chronic pain is associated with worse health and substance use treatment outcomes. This claim should be cited.

RESPONSE: The four references in the sentence following that one relate to poorer health (2 references) and enduring substance misuse problems (2 references). 
6.) In several instances, the authors state that "patients are associated" with a number of outcomes. These sentences should be edited so that it is clear that the presence of chronic pain is associated with a number of outcomes...not the patients themselves.

RESPONSE: We have now been through this section and revised the text where applicable, in accordance with this reviewer's comment.

7.) The authors make a relatively vague argument that previous studies have focused on substance use as a binary variable or illicit opioid use versus any other substance use. Although this statement is true, this argument needs to be strengthened as it is unclear to me what the authors are attempting to argue in terms of the novelty of their study. Furthermore, I think it's important for the authors to note that Trafton et al., 2004 Treatment needs associated with pain in substance use disorder patients: implications for concurrent treatment and Dunn, 2014 - Severity and interference of chronic pain in methadone-maintained outpatients found that participants with pain reported higher rates of marijuana, benzodiazepine, and sedative use. The aforementioned statement should be edited to reflect the findings of these studies.

RESPONSE: We have now revised this section and stated more clearly that we believe it is important to understand misuse of specific substances, rather than simply any substance misuse versus no substance misuse. We believe that this now provides stronger support of our objective of examining specific drug misuse.

The Dunn and Trafton articles have now been discussed ( $\mathrm{p} 5$ ).

8.) On Page 5, the authors state that "persistent pain was associated with poorer outcomes at 12-month follow up" in the Caldeiro, 2008 study. More detail should be provided. Which outcomes were poorer?

RESPONSE: We have clarified that this refers to assessment of abstinence. 
9.) Is there any evidence that can be cited to support the claim that initiation of illicit drug use after commencing treatment is a more severe negative outcome compared to continuation of drug use despite treatment?

RESPONSE: We are not aware of any literature that might support this assertion. In consequence, we have reworded this at the end of the Introduction section and in the Results section. It now reads, 'Continuation and initiation of illicit drug use were considered separately from each other since initiation of illicit drug use after commencing treatment may present different clinical challenges compared with continuation of illicit drug use despite treatment'.

Methods

10.) I have several questions related to how self-reported substance misuse was assessed. In the Introduction, the authors state that substance misuse will be compared between groups at 5 -year follow up. In the Methods section, the presence of substance misuse is defined as three positive test results in a 12-month period. It is unclear to me whether substance misuse was assessed only in the 12-month period preceding the 5-year follow-up or whether substance misuse was assessed throughout the 5-year follow-up period. Clarification would be helpful.

RESPONSE: Thank you for highlighting this lack of clarity. We have now added information to the Methods section explaining that self-reported substance misuse was recorded at study inception only, and that urinalysis data were obtained from the 12-month period at study inception (2005) and from the 12-month period at 5-year follow-up.

\section{1.) What diagnostic criteria were used to diagnose opioid dependence?}

RESPONSE: We have now clarified that the World Health Organisation's International Classification of Diseases (ICD v9) was used to diagnose opioid dependence.

\section{2.) It might be helpful if the authors provided a breakdown of how many participants} were dependent on heroin versus prescription opioids.

RESPONSE: We agree this would be interesting; however, the specific breakdown was not available from the service. The information provided by the service was that there is a prevalence of around $80 \%$ of patients entering treatment for heroin addiction. 
13.) How was the duration of pain assessed? The BPI-SF does not have any questions assessing duration.

RESPONSE: A question was added to the BPI-SF, asking patients to state (in months or years and months) the duration of their pain at study inception. This information has now been added to the Materials section.

14.) The authors should consider editing the Procedure section of the Methods to make it clear that urinalysis results are the primary outcome (as opposed to self-reported substance use).

RESPONSE: This section has now been edited to reflect the relative importance of the urinalysis results within the study.

15.) This is extremely minor but one reference (21) is still presented as a superscript. This should be changed to match the other references included in the manuscript.

RESPONSE: Thank you for pointing this out; it has now been corrected.

16.) In the present study, illicit drug use was defined as at least three positive test results in a 12-month period. This seems to be a relatively conservative estimate of illicit drug use. Is there a precedent in the literature for this definition?

RESPONSE: We have not encountered a precedent for this definition. There is no agreed definition of illicit drug use based on number of positive urine tests. Our choice was based on our recognition of the high risk of false positives and advice from an addiction psychiatrist. We have encountered a precedent for examining percentage of positive drug screens; however, we did not feel that that was as relevant as understanding prevalence of misuse. We agree that this may have resulted in a conservative estimate of illicit use, and were deliberately conservative to ensure that we did not include patients with false-positive results. 
Results

17.) Baseline differences between the two groups should be referenced in the Limitations paragraph of the Discussion. Is there a discernible self-selection bias? Is the generalizability of these findings limited in any way?

RESPONSE: The only significant demographic group difference was age (35 vs 32 ) and this is now noted in the Limitations section.

18.) For some of the between-group comparisons (e.g., absence from work due to illness) the $\mathbf{n}$ is quite small. This should at least be noted in the Limitations section of the Discussion.

RESPONSE: As per Reviewer \#1's suggestion, this section has been reduced substantially. In consequence, there are no longer any variables that have a small number of participant responses.

19.) Is it possible that participants were not retained in treatment because they improved and no longer needed services? Given that the reasons for non-retention are often unclear, particularly over the course of a 5-year follow-up, I'm not sure that the retention statistics provide a great deal of useful information.

RESPONSE: We accept this point and, in consequence, have removed table 3 . The text now simply reports the number retained in treatment and the proportion of each group that died (to highlight the potential survivor bias).

20.) Was pain status assessed at the 5 -year follow-up. It's plausible that the pain status may have changed for some individuals. Because the follow-up period is so long, a person who began to experience chronic pain symptoms mid-way through the follow-up period would fall into a new category by the endpoint.

RESPONSE: Unfortunately, as a result of limited resources, we were unable to assess pain at follow-up. We agree that this is a key limitation of our work. In consequence, we have now addressed this at the beginning of the Limitations section. 
21.) In the third paragraph of the Discussion, the authors introduce findings that were not presented in the Results section pertaining to rates of heroin use at entrance into the program and over the course of the study. Even if the authors choose not to include this data in a table, this information should be presented sooner, preferably in the Results section.

RESPONSE: These data have now been included in Table 1.

22.) The authors should cite studies that support their claim that psychiatric problems are more prevalent among opioid-dependent patients with pain problems (E.g, Dhingra et al., 2012 - Epidemiology of pain among outpatients in methadone maintenance treatment programs and Fox et al., 2012 - Pain is not associated with worse office-based buprenorphine treatment outcomes).

RESPONSE: This has now been referenced, as suggested.

23.) The authors spend an inordinate amount of time discussing the fact that psychiatric comorbidities may explain their findings. Given that the authors did not assess psychiatric symptoms for the purpose of the present study, this explanation seems speculative. Although this explanation is certainly worthy of mentioning, I think the authors might be better off focusing on issues that can be directly tied to the data collected for the purpose of the present study.

RESPONSE: We considered the possible link to psychiatric co-morbidities in just 4 sentences, as one possible explanation for some of our findings. We cited 9 references to support this consideration, so do not believe that we were being speculative. We are happy to take editorial advice on whether this section should be reduced. The rest of the Discussion generally focuses on findings that can be tied directly to our data, and/or to other possible explanations for, and implications of these. 
24.) The Limitations section of the Discussion needs work. I can think of a number of other limitations that are worthy of inclusion: 1.) the use of only two time points as opposed to looking at substance use over multiple time points during the 5 -year interval, 2.) issues of generalizability mentioned in Comment 16, and 3.) power issues mentioned in Comment 17.

RESPONSE: The Limitations section has now been rewritten, in light of this and other reviewer's comments. Thank you for the suggestions. 